\title{
Inducible cAMP Early Repressor (ICER) and Brain Functions
}

\author{
Gilyana Borlikova • Shogo Endo
}

Received: 4 February 2009 /Accepted: 7 April 2009 /Published online: 13 May 2009

(C) Humana Press Inc. 2009

\begin{abstract}
The inducible cAMP early repressor (ICER) is an endogenous repressor of cAMP-responsive element (CRE)mediated gene transcription and belongs to the CRE-binding protein (CREB)/CRE modulator (CREM)/activating transcription factor 1 (ATF-1) gene family. ICER plays an important role in regulating the neuroendocrine system and the circadian rhythm. Other aspects of ICER function have recently attracted heightened attention. Being a natural inducible CREB antagonist, and more broadly, an inducible repressor of CRE-mediated gene transcription, ICER regulates long-lasting plastic changes that occur in the brain in response to incoming stimulation. This review will bring together data on ICER and its functions in the brain, with a special emphasis on recent findings highlighting the involvement of ICER in the regulation of long-term plasticity underlying learning and memory.
\end{abstract}

Keywords ICER - CRE-mediated gene transcription . CREB · Long-term plasticity · Epileptogenesis · Learning · Memory $\cdot$ cAMP $\cdot$ cAMP-dependent $\cdot$ Immediate early gene

\section{Introduction}

Regulation of gene transcription through cAMP-responsive element (CRE)-mediated mechanisms is one of the important ways an organism and the brain adapt to ever changing environments. Transcription factors from the CRE-binding protein (CREB)/CRE modulator (CREM)/activating transcription factor 1 (ATF-1) gene family bind to CREs in

G. Borlikova $\cdot$ S. Endo $(\bowtie)$

Unit for Molecular Neurobiology of Learning and Memory,

Okinawa Institute of Science and Technology,

Uruma, Okinawa 904-2234, Japan

e-mail: sendo@oist.jp promoter regions of different genes and mediate the response of the cell to extracellular stimuli [1-5]. CREB was the first CRE-binding factor to be characterized [6,7]. Numerous studies have established a strong connection between CREB and neuronal plasticity [8-11]. However, the outcome of CRE-mediated gene transcription does not solely depend on CREB binding, but on the competitive binding of several dimerized transcription factors, including activators and repressors of gene transcription. Among the members of the CREB/CREM/ATF-1 gene family, the inducible cAMP early repressor (ICER) is unique in that it is not only a potent endogenous repressor of CRE-mediated gene transcription, but also is highly inducible by a variety of stimuli $[12,13]$. In contrast, most other members of the family are noninducible and are ubiquitously expressed [14-16].

Until recently, the main interest in ICER in the field of neuroscience was connected to its role in regulation of the circadian rhythm and neuroendocrine function [17-19]. However, other roles of ICER have recently come under the spotlight, shifting attention to its potential role in the regulation of neuronal plasticities that underlie higher nervous functions, such as learning and memory in particular [20,21].

\section{ICER Gene Structure and Regulation}

\section{Gene Structure}

ICER is a product of the CREM gene, and ICER messenger RNAs (mRNAs) are transcribed through an alternative intronic promoter (P2) $[12,13]$. The CREM gene contains multiple exons and gives rise to several alternative splicing variants that encode both transcriptional activator and repressor proteins (Fig. 1a) [22, 23]. One of the several interesting features of the CREM gene is that it possesses 
two DNA-binding domains (DBD I and DBD II), only one of which is included into the final protein.

Transcription from the constitutively active $\mathrm{P} 1$ promoter yields CREMs. CREMs share extensive homology with CREB: a DBD in the carboxyl terminus, and a kinaseinducible domain (P-Box) and a $\gamma$-domain (absent in CREM $\gamma$ ) in the amino terminus (Fig. 1a). CREM $\tau$, in addition contains glutamine-rich activation domains (Q1 and Q2).

ICER, on the other hand, is transcribed from the internal P2 promoter (Fig. 1b). ICERs contain a short and conserved $\mathrm{N}$-terminal sequence followed by a $\gamma$-domain (absent in $\gamma$-isoforms) and one of the DBDs, thus encoding small proteins with the predicted molecular weight of 12 and $13.5 \mathrm{kDa}(\sim 15$ and $\sim 19 \mathrm{kDa}$ in SDS-PAGE). The two DBDs encoded by the CREM gene are composed of basic and leucine zipper domains, which are involved in both dimerization and DNA binding of CREM and ICER [12, 13, 22].

\section{Isoforms}

Alternative splicing of the ICER transcript results in four different isoforms: ICER I, ICER I $\gamma$, ICER II, and ICER II $\gamma$ (Fig. 1b). ICER I isoforms contain DBD I, while ICER II isoforms contain DBD II. The two $\gamma$ isoforms (ICER I $\gamma$ and ICER II $\gamma$ ) lack exon $\gamma$. ICER I mRNA contains sequences encoding both DBD I and II, but the stop codon located in the carboxyl terminus of DBD I prevents insertion of DBD II into the protein [23]. All ICER isoforms homodimerize or heterodimerize with other members of the CREB/CREM/ ATF-1 family [12, 13, 24, 25].

\section{Repressor Activity}

ICERs lack activation domains and the kinase-inducible domain (Fig. 1), but the DBD allows their homo- or heterodimerization and binding to CRE elements. The lack of a transactivation domain makes ICERs potent repressors of cAMP-induced transcription of CRE-containing genes. However, not much is known at the moment about the levels of ICER required to suppress CRE-regulated transcription in vivo. In cultured pinealocytes, co-transfection with ICER completely abolishes cAMP-mediated induction of various CRE-containing reporters, starting at substoichiometric concentrations [13]. Co-transfection of a promoter-luciferase reporter construct of corticotrophin releasing hormone (CRH, also referred as CRF) with ICER I cDNA did not significantly affect basal $\mathrm{CRH}$ promoter activity, but dose-dependently inhibited forskolinstimulated promoter activity [24]. More experiments are required to determine the effectiveness of ICER's transcription repressor activity in the nervous system.

Regulation

The promoter of ICER (P2) contains a cluster of four CRElike cAMP autoregulatory elements (CAREs) organized in two tandems, CARE1-CARE2 and CARE3-CARE4. These CAREs are strongly inducible and are recognized by a variety of CRE-binding proteins, including CREB. Phosphorylated CREB binds to CAREs in the P2 promoter and rapidly activates ICER expression [12]. After induction, ICER can compete with CREB for the ICER promoter,

a

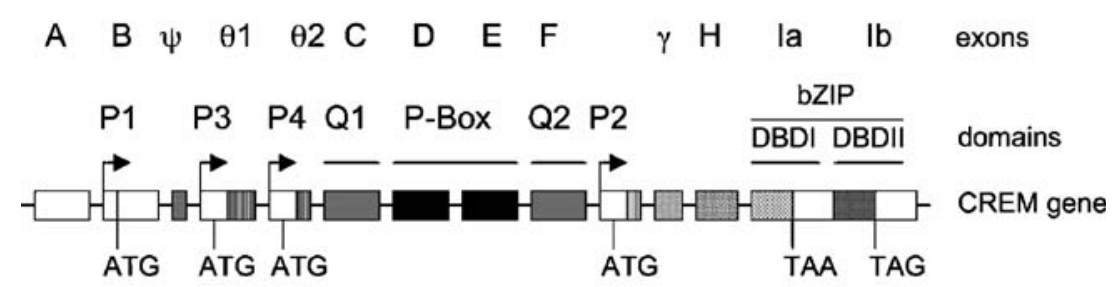

b

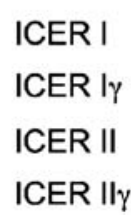

Fig. 1 Schematic representation of the gene encoding CREM/ICER. a The intron/exon structure of the CREM/ICER gene. Also shown are CREM promoters (P1, P3, and P4); ICER promoter (P2); glutaminerich domains (Q1 and $\mathrm{Q} 2)$; kinase-inducible domain (P-Box); and DNA-binding domains (DBD I and DBD II) of the ICER gene, containing leucine zipper and basic regions (bZIP). $A T G$, initiation

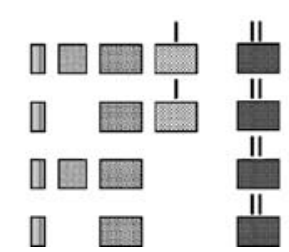

methionine; TAA and TAG, stop codons. b Schematic mRNA structure of four ICER isoforms and corresponding exons. ICER I mRNA contains sequences encoding both DBD I and II, but the stop codon located in the carboxyl terminus of DBD I prevents insertion of DBD II into the protein 
thus suppressing its own transcription, which in effect constitutes a negative autoregulatory loop [12]. This negative feedback loop serves as a temporal gene controlling mechanism that allows the cAMP-dependent signaling cascade to prepare for subsequent incoming signals [26, 27].

Different binding affinities of the different CAREs for CREB, CREM, and ICER proteins together with different transcription-driving efficiencies of the CAREs provide additional options for fine gene regulation $[12,28]$. Moreover, ICER expression can be activated in a nonCRE-dependent manner [29]. Further characterization of the relationships between different extracellular stimuli, intracellular cascades, and resulting ICER activation will help to advance our understanding of the "fine-tuning" of transcription in response to differential stimulation.

Intracellular levels of ICER protein are controlled by transcription regulation and by protein degradation through the ubiquitin-proteasome system [30]. Different ICER isoforms have different half-lives: ICER I is the most stable followed by ICER II, whereas $\gamma$-isoforms are short-lived, with a half-life of $\sim 3 \mathrm{~h}$ [30]. The different half-lives of different isoforms may contribute to the temporal regulation of CRE-dependent gene transcription during the course of physiological phenomena.

\section{ICER Distribution and Induction Factors in the Brain}

ICER is expressed at uniformly low levels in the central nervous system, with the exception of some neuroendocrine structures, such as the pineal gland and hypothalamic nuclei, and sensory input and relay nuclei including the olfactory bulb and sensory brain stem nuclei [13, 18, 29, 31-33]. However, drastic upregulation of ICER expression has been demonstrated in response to a variety of stimuli [26].

The well-studied signal transduction pathway regulating ICER transcription is the adenylate cyclase-cAMP-cAMPdependent protein kinase (PKA)-CREB-ICER pathway. Numerous studies have demonstrated ICER induction in a variety of cell cultures after treatments that increase intracellular cAMP and induce PKA activation [12, 13, 24, 28, 34, 35]. ICER can also be induced through PKAindependent pathways such as the Ras-dependent nerve growth factor (NGF) pathway and the Janus kinase/signal transducer and activator of transcription pathway, the protein kinase $\mathrm{C}(\mathrm{PKC})$ pathway, as well as other pathways [26, 28, 29, 36]. Interestingly, some cell culture studies showed that histone deacetylase (HDAC) is required for the induction of ICER expression [36, 37]. Application of brain-derived neurotrophic factor (BDNF) and dopamine to neuronal primary cultures also increases ICER levels $[29,35]$.
In Vivo Induction

Seizure activity strongly induces ICER. Electroconvulsive shock upregulates ICER mRNA in rat cortex and hippocampus [38], as do kainate-induced seizures [39], prolonged pilocarpine-induced seizures [40], and amygdala kindling stimulation [21]. Both agonists and antagonists of glutamate receptors induce ICER mRNA expression [39, 41-43].

Different types of stress, as well as CRH injections, increase ICER expression in a variety of neuroendocrine structures as well as in the striatum [12, 44-46]. Antidepressant and amphetamine treatments also upregulate ICER expression [46, 47], as do lithium chloride and nociceptin injections [48-50].

Purely physiological stimulation, such as exposure of dark-reared rats to light [39] and exploration of enriched environments [31, 32], also have the capacity to substantially upregulate ICER expression. Moreover, ICER mRNA is upregulated not only immediately after fear conditioning training but is also upregulated specifically when mice are presented with the conditioned stimulus (tone or context) $24 \mathrm{~h}$ later [21].

The ICER induction observed in response to a variety of physiological and non-physiological stimuli suggests that it may play an important role in restricting/suppressing responses to environmental stimuli. Thus, ICER may serve as a filter ensuring that only important, strong, or persistent incoming stimulation is transduced into plastic changes of the nervous system.

\section{Time Course of Induction}

ICER is classified as an early response gene [12]. Studies using different types of stimulation have demonstrated that ICER induction is relatively slow compared to the induction time course of other immediate early genes (IEGs; ICER expression peaks around 2-6 h after stimulation, depending on types of stimulation and cell types). Once induced, however, ICER upregulation lasts longer than the upregulation of other IEGs in the cell (often more than $24 \mathrm{~h})[24,35,39,41,51-54]$.

Amphetamine injection or restraint stress increases ICER mRNA levels in the striatum, reaching maximum levels around 3-4 h later followed by slow return to control levels within $12 \mathrm{~h}[25,46]$. Kindling stimulation causes a sharp increase in $c$-fos mRNA followed by rapid decline [21]. On the other hand, kindling increases ICER mRNA levels $1 \mathrm{~h}$ post-stimulation, which remains above basal levels for more than $6 \mathrm{~h}$ thereafter [21]. After pilocarpine-induced prolonged seizures, ICER mRNA reaches maximum levels around $6 \mathrm{~h}$, and then gradually declines; ICER protein levels are upregulated longer than $24 \mathrm{~h}$ after seizures [29]. During exploration of a novel enriched environment, the 
increase in c-Fos protein levels reaches maximum levels after $1 \mathrm{~h}$ and then declines [32]. By contrast, induction of ICER reaches a maximum around $6 \mathrm{~h}$ after exploration and remains upregulated even after 5 days of exploration [32].

\section{ICER as a Transcription Regulator}

The delayed and prolonged time course of ICER induction suggests that, after reaching a sufficient concentration in the nucleus, ICER acts to suppress gene transcription of other IEGs and/or their target late-response genes and eventually switches off the CRE-mediated gene transcription initiated by the original stimuli $[1,4,18,25,26,32]$. This is supported by the observations that ICER attenuates c-fos mRNA expression in cultured cells [28, 55]; that increase in ICER expression after nociceptin injection coincides with decreased c-Fos protein expression [48]; and that kindling stimulus-induced c-Fos protein expression is attenuated by ICER overexpression in vivo [21]. Further support is provided by findings that herpes simplex virus (HSV)ICER injections into the rat nucleus accumbens repress amphetamine-induced expression of BDNF [46] and upregulation of ICER reduces expression of the $\alpha 1$ subunit of gamma-aminobutyric acid $(\mathrm{GABA})_{\mathrm{A}}$ receptors in primary neocortical cell cultures [35]. Thus, ICER may serve as an important factor in shutting down cAMP-inducible gene transcription [32], together with the inactivating of phosphorylated CREB by dephosphorylation [5].

Being an inducible and powerful repressor of cAMPdependent transcription, ICER can potentially affect the expression of numerous CRE-containing genes, including genes whose products are critically involved in neuronal plasticity; genes that encode transcription factors (c-fos, JunD, Krox-20, Krox-24, creb, icer); genes that encode neurotransmission-related proteins (enkephalin, galanin, somatostatin, $\mathrm{CRH}$, tyrosine hydroxylase, $\mathrm{GABA}_{\mathrm{A}}$ receptor subunits, $\beta$-adrenergic receptor subunits, inducible nitric oxide synthase); and genes that encode growth factors, such as $\operatorname{BDNF}[1,5,26]$. In addition, ICER can regulate transcription by binding to sequences other than CRE (e.g., activator protein 1, AP1) [26].

As different ICER isoforms utilize different DBD domains, by analogy with other CREM isoforms, they may have different affinity for heterodimerization with other members of the CREB/CREM/ATF-1 gene family [22, 23] and different CRE affinity [35]. For example, ICER I is slightly more potent than ICER II $\gamma$ in suppressing endogenous and forskolin-stimulated $\mathrm{CRH}$ promoter activity in cultured cells [24]. However, Misund and colleagues did not find any substantial difference in the repression of CRE-driven transcription between the ICER I and II $\gamma$ isoforms in human embryonic kidney (HEK) 293 cell variants with controllable overexpression of these isoforms [55]. The authors hypothesized that the putative different biological functions of the ICER isoforms may instead be related to differences in their expression levels in response to different types of stimulation or in different cell types.

In addition to the regulation of CREB-dependent transcription at the level of competition for CRE sites, ICER is proposed to regulate CREB at the level of protein stability, since in cell cultures co-transfected with CREBand ICER-containing vectors, ICER significantly decreases the intracellular levels of exogenously expressed CREB [56]. Thus, ICER may regulate CRE-mediated transcription in several different ways. Together with the already mentioned potential differential affinity of the four ICER isoforms toward particular dimerization partners and different CRE sites, as well as tissue/cell-type specificity, this simultaneous versatility and variability gives ICER the potential to play very important roles in "fine-tuning" CRE-mediated transcription according to different incoming information. The final outcome may be further affected by interactions occurring between two binding events: ICER binding to CREs and transcription activator binding to other sites [34]. All these aspects warrant careful further investigation, specifically in application to neurons.

\section{Role of ICER in Circadian Regulation}

In neuroendocrine tissues, ICER is the most abundant of all CREM isoforms [12, 13, 15, 44]. ICER plays an important role in the neuroendocrine system, regulating melatonin synthesis during the course of the circadian rhythm [19, 57] and coordinating reaction to hypothalamic-pituitary-adrenal (HPA) axis stimulation [44, 45]. The role of ICER in circadian regulation has already received much attention and is summarized in a series of excellent reviews [17-19, 58].

\section{Role of ICER in Apoptosis}

ICER plays an important physiological role in apoptosis in the nervous system [26, 39, 59-63]. Stimuli evoking neuronal cell death in the brain upregulate endogenous ICER expression [39]. Endogenous ICER expression is also upregulated in a variety of cell cultures undergoing apoptosis [59-62]. Moreover, both adenoviral vectordriven overexpression of ICER II $\gamma$ and cell transfection with any of the four ICER isoforms also result in apoptosis [39, 41, 59, 60, 62]. While all four ICER isoforms are induced after pro-apoptotic treatment, isoforms (I $\gamma$ and II $\gamma$ ) 
lacking the $\gamma$ domain show the strongest induction [60, 62]. ICER might promote apoptosis through downregulation of the anti-apoptotic gene $b c l-2$ [59, 62, 64]. ICER may interfere with the pro-survival action of $\mathrm{CREB}$ and contribute to neurodegeneration from neurotoxicity, trauma, and trophic deprivation or disorder [62]. However, studies employing in vivo ICER overexpression did not report any signs of neuronal cell death [20, 21, 46]. Recent findings [62] suggest that in the intact brain the pro-apoptotic action of ICER may be more tightly regulated and less pronounced [21], thus explaining the discrepancy between the in vitro and in vivo findings.

\section{ICER and General Behavior}

Data regarding involvement of ICER in the regulation of basic behavioral functions are sometimes contradictory, reflecting differences between ICER manipulating procedures (viral vector transfer or chronic genetic manipulation) and animal species used (mice or rats). Overall, the accumulated results suggest that ICER may play different roles depending on the site of manipulation (brain structure) and type of behavior (Table 1).

ICER-specific overexpressing (OE) or knockout (KO) mice display normal locomotor activity in the home cage and open field [21]. Moreover, overexpression of ICER in hippocampus does not lead to changes in rat locomotor activity [20]. On the other hand, overexpression of ICER in the nucleus accumbens decreases the spontaneous activity of rats in the open field but strongly potentiates amphetamine-induced locomotor activity [46]. Characterization of CREM/ICER-KO mice (KO mice lacking all CREM isoforms including ICER) yielded contradictory results that might stem from differences in genetic background and experimental procedures used. For example, Maldonado and colleagues observed significantly increased spontaneous locomotor activity in mutant animals; moreover, mutant animals displayed homogeneously high activity during both light and dark cycle periods [65]. By contrast, Conti and colleagues observed no differences in basal locomotion in the home-cage activity of mutant and wild-type mice [47].

Overexpression of either ICER I or II in the entire forebrain does not affect mouse behavior in the elevated plus-maze test [21], while overexpression of ICER in the nucleus accumbens produces robust anxiogenic-like effects and increases "neophobia" in rats [46]. CREM/ICER-KO mice show decreased anxiety in the elevated plus- and zero-maze tests [65]. ICER-specific KO mice do not show changes in the elevated plus-maze; however, longer latencies to enter the light compartment during the light/dark transition test suggest that these mice have increased anxiety [21].
Overexpression of ICER in the nucleus accumbens increases responsiveness to natural rewards such as familiar sucrose and social interaction, and produces antidepressantlike effects in the forced swim test [46]. On the other hand, CREM/ICER-KO mice show similar levels of immobility compared to wild-type mice in the forced swim and tail suspension tests [47].

\section{ICER, Stress, and Depression-like Behavior}

Stress induces ICER expression in different parts of the HPA axis, and ICER regulates transcription of the CRH gene during stress [24, 25, 45]. Under normal conditions, stimulation of the cAMP cascade by stress produces a dual effect: first facilitating $\mathrm{CRH}$ gene transcription via phospho-CREB, and later inhibiting it via induction of ICER. Thus, ICER activation, by suppressing CRH transcription, serves as a protective mechanism helping to avoid the consequences of excessive expression of $\mathrm{CRH}$ $[24,25,45]$.

Decreased depression-like behavior in ICER-overexpressing rats [46] may, in part, result from reduced CRH production, as the overproduction of $\mathrm{CRH}$ is believed to contribute to depression [66, 67]. ICER may also be a critical molecular mediator of tricyclic antidepressant action, as suggested by its strong induction in response to desipramine treatment [47]. Although ICER is not essential for the behavioral antidepressant effect of the drug, it is critical for desipraminemediated reduction of stress-induced plasma corticosterone levels [47].

The results obtained through ICER gene manipulations may provide important clues that will help to reveal the precise molecular mechanisms underlying chronic stress states and will contribute toward development of novel antidepressant drugs.

\section{ICER and Regulation of Epileptogenesis}

Seizures alter the activity of the CREB/CREM/ATF-1 gene family and their target genes. Kindling is widely studied as a model of epileptogenesis and as a form of neuroplasticity [68-70]. Furthermore, kindling development requires gene transcription and translation [68, 71]. The term kindling refers to the phenomenon that periodic mild electrical stimulation of one of many brain sites at stimulus intensities initially too low to produce any effects eventually leads to the development and gradual intensification of elicited motor seizures [72]. CRE binding of CREB is transiently upregulated after kindling stimulation [73]. As mentioned previously, ICER mRNA levels are increased in the hippocampus and other brain regions after seizures provoked 


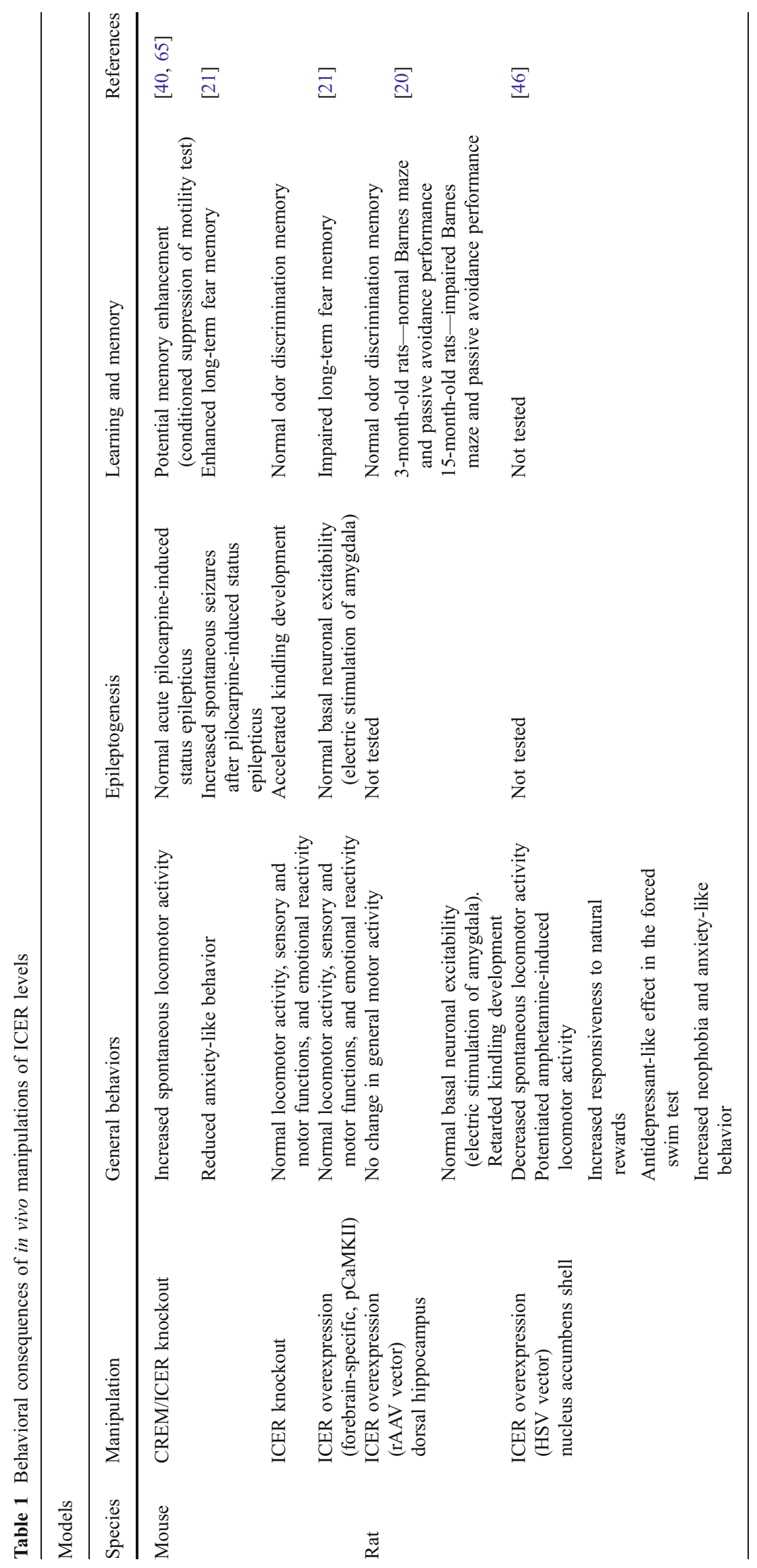


by kindling stimulation [21], electroconvulsive shock [38], kainate [39], and pilocarpine [40].

The finding that CREM/ICER-KO mice develop more severe epilepsy after pilocarpine-induced status epilepticus (SE) than non-mutant littermates supports a close tie between ICER and the genesis of seizures [40]. Furthermore, using ICER-specific mutant mice (both ICER-OE and ICER-KO mice) we have recently shown that ICER negatively regulates epileptogenesis: Overexpression of ICER leads to the retardation of kindling development, while lack of ICER significantly facilitates kindling [21]. These anti-epileptic effects of ICER fit nicely with the data showing that prolonged hyperactivation of CREB-mediated gene transcription leads to sporadic epileptic seizures [74]. The increased ICER levels may achieve the anti-epileptic effect by restricting and suppressing excessive CREdependent transcription. On the other hand, the removal of ICER from the system compromises endogenous suppression of CRE-dependent gene activation and results in aberrant synaptic plasticity and seizure development [21, 40].

Pilocarpine-induced SE upregulates ICER, which binds to the CRE site in the Gabral promoter and decreases expression of $\mathrm{GABA}_{\mathrm{A}}$ receptor $\alpha 1$ subunits [29]. This leads to a decrease in the number of $\mathrm{GABA}_{\mathrm{A}} \alpha 1 \gamma 2$-containing receptors in the dentate gyrus of hippocampus, potentially promoting network disinhibition [29, 35]. A similar process occurs in hippocampal cell cultures [35].

The apparent discrepancy of the above findings might be due to differences between the effects of constitutive changes in ICER levels (in the case of gene manipulations) and the effects of transient ICER upregulation (in the case of intact systems). Another possible explanation is that the effects of whole forebrain (or brain) changes in ICER levels differ from those of region-specific changes in ICER levels. Further studies are needed to better understand the role of ICER in epilepsy, but even in the current state, all these findings clearly show that ICER does play an important role in the regulation of epileptogenesis.

\section{ICER and Memory}

The cAMP-PKA-CREB system is evolutionarily well conserved $[5,11]$. The indispensable role of CREB and CRE-mediated gene transcription in neuronal plasticity underlying learning and memory has been established in a variety of animal species [75-79]. Manipulation of CREB levels or activity is considered to be a promising way to study and, eventually, improve memory $[10,80]$. However, studies to date have yielded mixed results [81-84], highlighting that manipulation of a constitutively expressed transcription factor is complicated by wide non-selective changes and produces compensatory changes in expression of other CREB/CREM/ATF-1 gene family members [8587]. Being a potent endogenous repressor of CREB, ICER is strategically poised to play an important role in memory formation. Its low basal level of expression and high inducibility in response to various types of stimulation suggest that ICER is an ideal potential target for memoryimproving manipulations. However, until recently, little attention has been given to the role of ICER in learning and memory.

Machado and colleagues proposed that ICER belongs to a subgroup of IEGs, called induced preferentially by depolarization-IEGs, that are more likely to play specific roles in synaptic plasticity than IEGs, which are induced by a number of factors in addition to depolarization [88]. Exploration of enriched environments upregulates ICER expression in the barrel-related columns of primary somatosensory cortex, an important site related to familiarization with novel environments and learning about new stimuli [31, 32]. Increased ICER mRNA levels are also detected in the amygdala after fear conditioning training and testing [21].

The overexpression of ICER in dorsal hippocampus through a recombinant adeno-associated virus (rAAV) vector did not cause 3-month-old rats to show changes in memory tests, which included a passive avoidance task and the Barnes spatial maze [20]. When the same animals were re-tested in these tasks at 15 months of age, ICERoverexpressing rats performed significantly worse than the sham-operated control rats of the same age. Although rAAV-mediated ICER overexpression was widespread and robust, it did not cover $100 \%$ of hippocampal neurons. Mouravlev et al. proposed that in young adult rats the remaining non-transfected neurons were sufficient to maintain normal function [20]. However, as the CREB system function declined with age, the same level of ICER overexpression became disruptive, as the non-transduced cells were no longer able to compensate.

To investigate the role of ICER in neuronal plasticity and memory in detail, we have generated two types of ICER mutant mice: ICER-overexpressing (ICER-OE) and ICERspecific knockout (ICER-KO) mice [21]. In accordance with the non-constitutive and inducible nature of ICER, specific overexpression or deletion of the gene did not cause any compensatory changes in the expression level of CREB or CREM isoforms. While both ICER-OE and ICER-KO mice were spared of any robust changes in locomotor activity, sensory functions, and emotional responses, they demonstrated specific changes in memory, as assessed using fear conditioning. After conditioning with two tone-foot shock pairs, the mice were sequentially tested for short-term $(1 \mathrm{~h})$ and long-term $(24 \mathrm{~h})$ tone-dependent 
fear memory, followed by long-term context-dependent fear memory (48 h). Overexpression of ICER led to impaired long-term tone- and context-dependent fear memory, leaving short-term memory unchanged (Fig. 2a). By contrast, deletion of ICER in ICER-KO mice-although inefficient in the case of the standard fear conditioning protocol (ceiling effect, Fig. 2b)-led to enhancement of long-term memory formation when a weak conditioning protocol (producing weak long-term memory in controls) was employed (Fig. 2c).
The results showing that overexpression of ICER specifically impairs long-term fear memory resemble those reported previously in mice in the case of CREB downregulation [75, 82, 89] or overexpression of dominant negative mutant CREB $[90,91]$ and are consistent with the role of ICER as an endogenous CREB antagonist. The disruption of CREB gene results not only in the impairment of long-term memory but also leads to the significant upregulation of several CREM isoforms, ICER in particular [86]. This raises the possibility that behavioral phenotypes

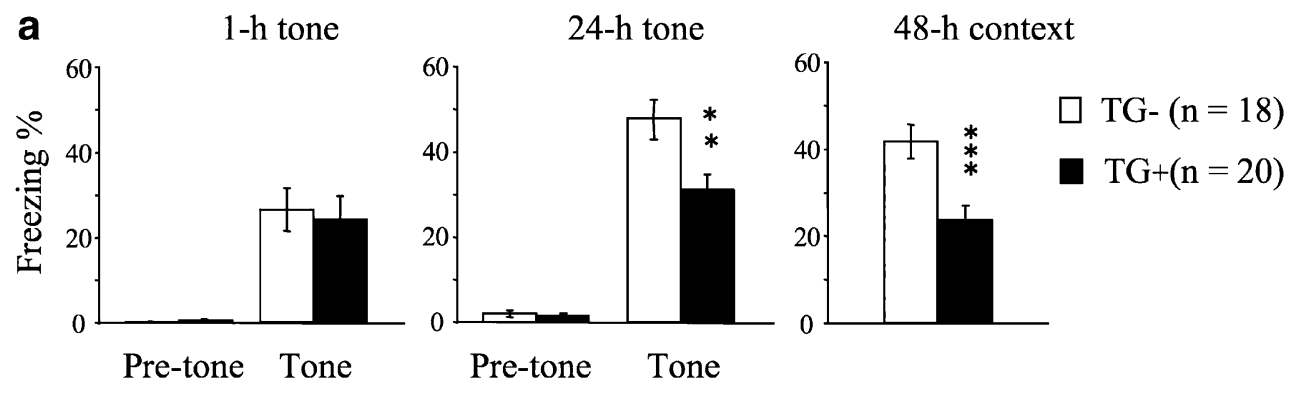

b

1-h tone
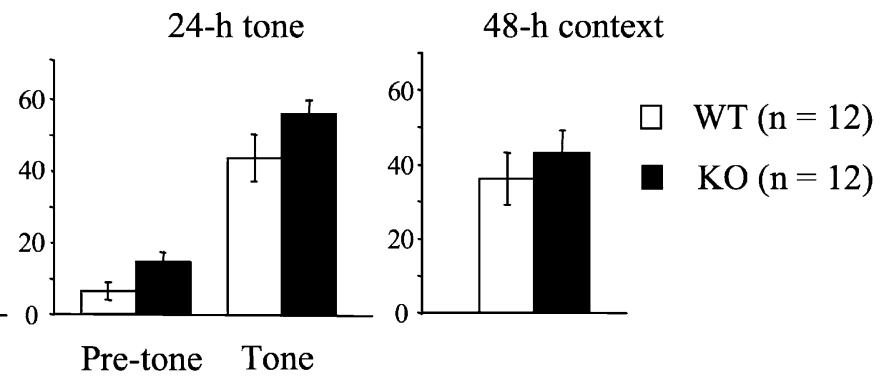

C

1-h tone

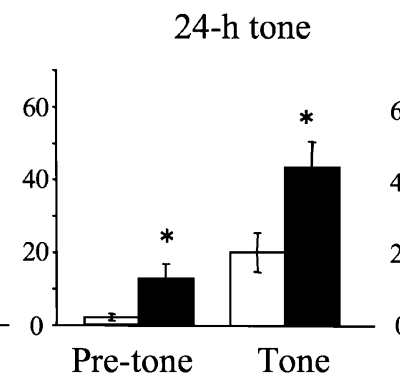

24-h tone
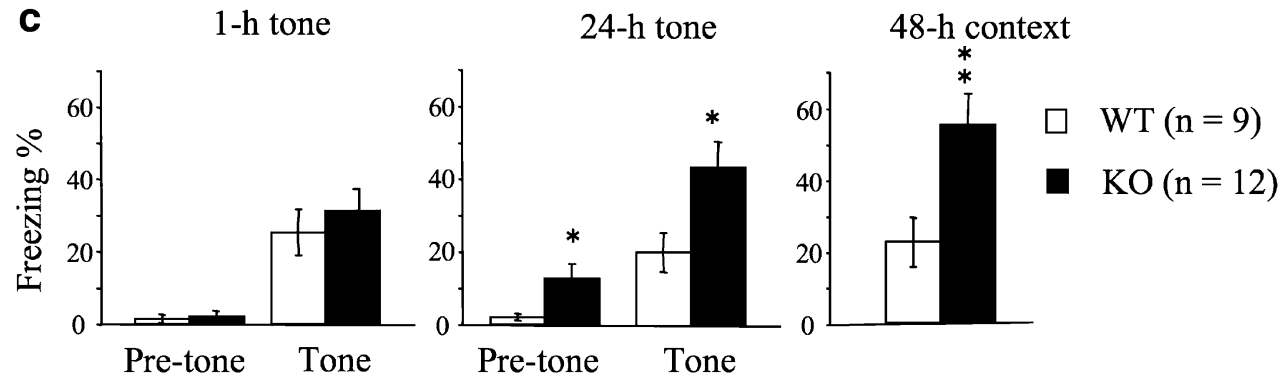

Fig. 2 Fear conditioning in ICER mutant mice. Conditioned freezing to tone and context was compared in ICER-overexpressing (OE) transgenic mice $\left(T G^{+}\right)$and their non-TG littermates $\left(T G^{-} ; \mathbf{a}\right)$, in ICER-knockout $(K O)$ mice and their wild-type $(W T)$ littermates after a standard conditioning protocol (b), and in ICER-KO mice and WT littermates after a weak conditioning protocol (c). a No difference was observed between non-TG mice and ICER-OE mice $1 \mathrm{~h}$ after conditioning ( $1 \mathrm{~h}$ tone). However, $24 \mathrm{~h}$ ( $24 \mathrm{~h}$ tone) and $48 \mathrm{~h}(48 \mathrm{~h}$ context) after conditioning, tone-dependent and context-dependent freezing were significantly attenuated in ICER-OE mice. b After being subjected to a standard conditioning protocol, ICER-KO mice and WT littermates exhibited similar freezing levels during the tone-dependent

tests performed $1 \mathrm{~h}(1 \mathrm{~h}$ tone) and $24 \mathrm{~h}(24 \mathrm{~h}$ tone $)$ after conditioning and during the context-dependent test (48-h context). c After being subjected to a weak conditioning protocol, there was no significant difference in conditioned freezing between ICER-KO and WT mice $1 \mathrm{~h}$ after conditioning ( $1 \mathrm{~h}$ tone). However, ICER-KO mice showed increased freezing during both pre-tone and tone presentation in the tone-dependent test performed $24 \mathrm{~h}$ after conditioning ( $24 \mathrm{~h}$ tone). Context-dependent freezing in ICER-KO mice was also enhanced ( $48 \mathrm{~h}$ context). Data are means $\pm \mathrm{SEM}$; number of animals per group are in parentheses. ${ }^{*} p<0.05 ; * * p<0.01 ; * * * p<0.001$ compared to non-TG or WT littermates (with modifications from [21]) 
of CREB-KO mice may not entirely result from the lack of CREB but, in part, may result from increased ICER levels. On the other hand, ICER-KO mice show enhanced fear memory, though only when a weak conditioning protocol is used. Again, these data are consistent with the results of CREB manipulations: In rats, the overexpression of CREB promotes the formation of long-term memory under training conditions that do not produce strong long-term memory among controls [92-94]. Taken together, the data show that both manipulations, the increase in the amount of transcription activator (CREB) or removal of transcription suppressor (ICER), change behavior in the same direction (i.e., enhance long-term memory).

Neither overexpression nor deletion of ICER affected memory tested in the odor discrimination task [21]. The role of ICER may be critical only for some types of memory; however, extensive data clearly demonstrate that CREB and CRE-dependent transcription play an important role in a wide array of memory tasks [75, 82, 95, 96]. It might also be the case that ICER-induced inhibition of transcription play a crucial role in "single-trial" or "subthreshold" learning, but more robust repeated training (as in an odor discrimination task) is able to overcome the effects of loss or overexpression of ICER. Further detailed examination of other types of memory is necessary in order to fully delineate memory systems critically dependent on CREB/ICER regulation.

\section{Further Possible Directions: ICER and Drug Addiction}

Through the occupation of CRE elements in the promoters of various critical genes and counteraction of CREBinduced activation, ICER has the potential to affect different types of higher brain functions. One of the promising directions to study is ICER's involvement in the regulation of reward learning and drug addiction. CREB is involved in regulation of drug reward [97-101], and overexpression of dominant-negative CREB increases cocaine reward [102, 103]. Involvement of ICER in the regulation of drug reward is supported by the data obtained using the psychostimulant amphetamine. Amphetamine administration increases ICER mRNA levels in the ventral striatum in a dose-dependent manner; this effect diminishes progressively with repeated drug administration [46]. In addition, overexpression of ICER in the nucleus accumbens strongly potentiates amphetamine-induced locomotor activity [46]. The same authors have found that overexpression of ICER also affects an animal's responses to natural rewards, leading to increased preference of familiar sucrose solution and increased social reward. Moreover, since both dopamine- $\beta$-hydroxylase and tyrosine hydroxylase genes contain CRE elements [104, 105], potentially, ICER may be able to modulate synthesis of dopamine as well as other catecholamines. Application of dopamine to primary rat neocortical cultures induces ICER activation and downregulates expression of the $\alpha 1$ subunit of $\mathrm{GABA}_{\mathrm{A}}$ receptor, leading to the downregulation of $\alpha 1$-containing $\mathrm{GABA}_{\mathrm{A}}$ receptors at the cell surface [35]. Hu et al. proposed that the dopamine neurotransmitter system uses the CREB signaling pathway to regulate inhibition in the brain, and ICER may be an important contributor to altered inhibitory processes in different pathological conditions, such as Parkinson's disease, brain trauma, and drug addiction [35].

\section{Conclusions}

Recent findings clearly show that ICER is involved in the regulation of plasticity underlying epileptogenesis and fear memory, as well as reaction to stress. However, further work is needed to assess ICER's involvement in plasticity underlying other types of learning and memory. The availability of genetically manipulated mice, the expression of ICER using virus vectors, and the conditional manipulation of ICER gene provide rich opportunities to dissect the physiological role of ICER in detail.

ICER acts as a negative regulator of long-term neuronal plasticity most possibly through suppression of plasticity-related gene expression (Fig. 3). As expression of ICER may be induced by the same stimuli and through the same molecular cascades that initiate formation of memory traces, ICER may play an overall positive role in the formation of adaptively meaningful learning and memory. By suppressing cAMP-induced transcription, ICER may serve as a filter that increases the "signal-to-noise ratio," sifts away weak stimulation, and allows only strong/ persistent information to gain access to long-term storage. However, the same filter might become counterproductive if the activating part of the process (e.g., CREB activity) weakens due to some circumstances. Accordingly, manipulations of ICER levels may, depending on the context, play both positive and negative roles in the organism.

In the neuroendocrine system, ICER induction by cAMP contributes to a refractory phase during which additional cAMP stimuli fail to elicit a full transcriptional response [106]. This mechanism has been proposed to make the transcriptional response to repetitive or prolonged stress dependent on the frequency or the duration of previous stress episodes [44, 106]. We would like to extend this further and speculate that, by the same mechanism, ICER may contribute to weaker memory formation after massed training procedures. In a similar framework, Won and Silva 


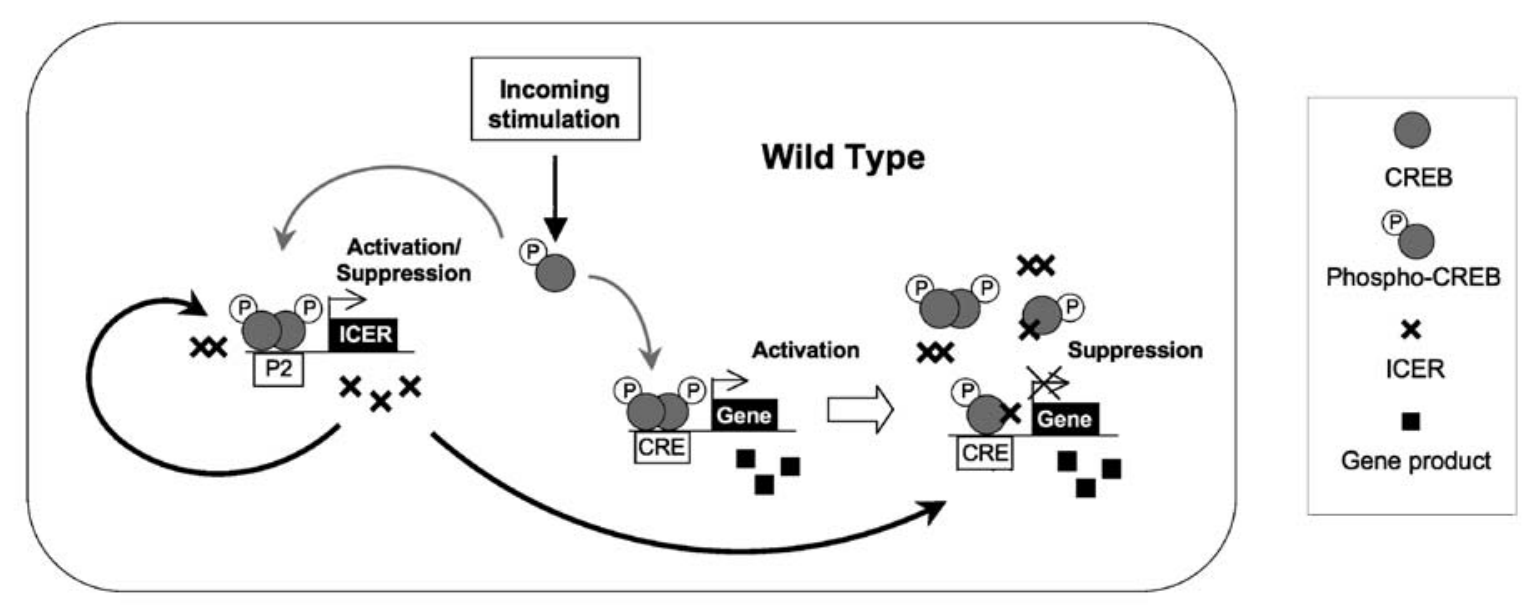

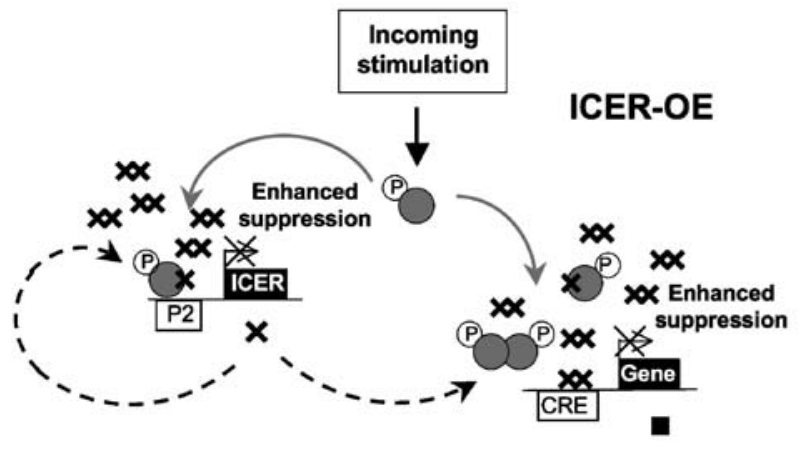

Fig. 3 Simplified schematic diagram of ICER acting as a regulator of long-term plasticity. In wild-type mice, incoming stimuli activate protein kinases and activated kinases phosphorylate CREB. Phosphorylated CREB activates CRE-containing gene transcription. Phosphorylated CREB also activates P2 promoter and initiates ICER transcription. ICER is a transcription repressor, and after reaching a certain concentration, starts to suppress CRE-dependent activation of gene expression, including its own expression (through the formation of ICER homodimers or ICER-CREB heterodimers that bind to CREs and block transcription). In ICER-OE mice, suppression of CRE-

have proposed that a refractory phase caused by ICER repression may play an important role in memory allocation in neuron networks, promoting dynamic memory allocation to different sets of neurons [107]. All these hypotheses position ICER as an important player in metaplasticity [108].

As we have tried to show, interesting data on ICER function in the brain have emerged recently. Overexpression of ICER, achieved through gene manipulation or viral vectors, is a powerful tool. However, while considering these results, one needs to be aware that the consequences of stable and long-lasting overexpression of ICER may be different from the consequences of the phasic, transient changes in gene expression that takes place under normal conditions in response to different incoming stimuli [46].

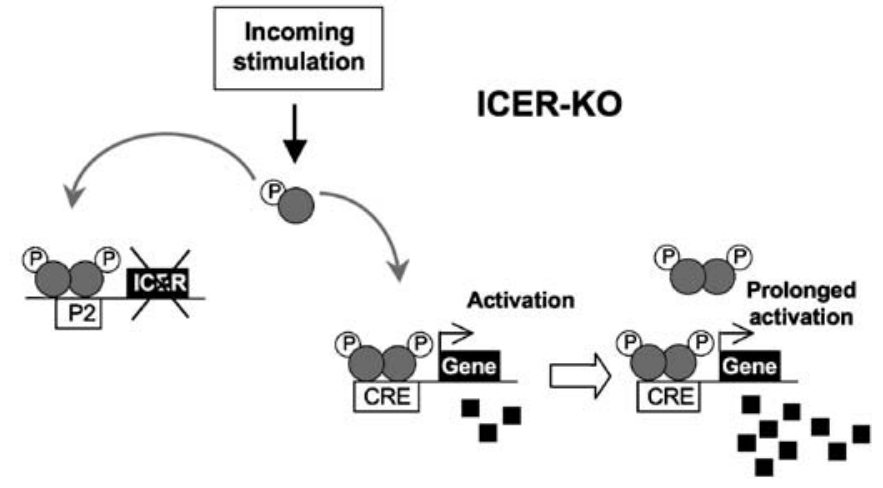

mediated gene transcription is enhanced by a constitutively available excess amount of ICER. In ICER-KO mice, lack of ICER-mediated suppression results in a prolonged activation of CRE-mediated gene transcription. Accumulated gene products that result from the transcription-translation of the gene are indicated as filled squares; ICER proteins are indicated as crosses; the transcription activator CREB is indicated by gray filled circles. P, phosphate group; P2, ICER promoter in the CREM/ICER gene; CRE, CRE-containing promoters (figure with modifications was adapted from [21])

But even with this constraint, the data obtained so far provide valuable insight into the role of ICER regulation of CRE-mediated transcription in brain function, firmly positioning it as an important "stimulus-transcription coupling" agent $[109,110]$. The data showing that ICER acts as a regulator of long-term memory formation and epileptogenesis suggest that the dynamic balance of CREB/ICER function is a crucial factor that determines which events will be fixed for long-term storage through neural plasticity. Further studies and a better understanding of the role of ICER in the regulation of long-term plasticity may contribute to the development of medications for various pathological conditions, such as posttraumatic stress disorder, drug addiction, epilepsy, and dementias. 
Acknowledgments The authors thank Dr. Nobuhiko Kojima for the critical reading of an early draft and for generating Fig. 3, and everyone who responded to the authors' inquiry regarding ICER and shared their recent findings.

\section{References}

1. Mayr B, Montminy M (2001) Transcriptional regulation by the phosphorylation-dependent factor CREB. Nat Rev Mol Cell Biol 2:599-609

2. Montminy M (1997) Transcriptional regulation by cyclic AMP. Annu Rev Biochem 66:807-822

3. Lee KA, Masson N (1993) Transcriptional regulation by CREB and its relatives. Biochim Biophys Acta 1174:221-233

4. Sassone-Corsi P (1995) Transcription factors responsive to cAMP. Annu Rev Cell Dev Biol 11:355-377

5. Lonze BE, Ginty DD (2002) Function and regulation of CREB family transcription factors in the nervous system. Neuron 35:605-623

6. Montminy MR, Bilezikjian LM (1987) Binding of a nuclear protein to the cyclic-AMP response element of the somatostatin gene. Nature 328:175-178

7. Hoeffler JP, Meyer TE, Yun Y, Jameson JL, Habener JF (1988) Cyclic AMP-responsive DNA-binding protein: structure based on a cloned placental cDNA. Science 242:1430-1433

8. Stevens CF (1994) CREB and memory consolidation. Neuron 13:769-770

9. Silva AJ, Kogan JH, Frankland PW, Kida S (1998) CREB and memory. Annu Rev Neurosci 21:127-148

10. Tully T, Bourtchouladze R, Scott R, Tallman J (2003) Targeting the CREB pathway for memory enhancers. Nat Rev Drug Discov 2:267-277

11. Frank DA, Greenberg ME (1994) CREB: a mediator of longterm memory from mollusks to mammals. Cell 79:5-8

12. Molina CA, Foulkes NS, Lalli E, Sassone-Corsi P (1993) Inducibility and negative autoregulation of CREM: an alternative promoter directs the expression of ICER, an early response repressor. Cell 75:875-886

13. Stehle JH, Foulkes NS, Molina CA, Simonneaux V, Pevet P, Sassone-Corsi P (1993) Adrenergic signals direct rhythmic expression of transcriptional repressor CREM in the pineal gland. Nature 365:314-320

14. Habener JF (1990) Cyclic AMP response element binding proteins: a cornucopia of transcription factors. Mol Endocrinol 4:1087-1094

15. Mellstrom B, Naranjo JR, Foulkes NS, Lafarga M, Sassone-Corsi P (1993) Transcriptional response to cAMP in brain: specific distribution and induction of CREM antagonists. Neuron 10:655665

16. Borrelli E, Montmayeur JP, Foulkes NS, Sassone-Corsi P (1992) Signal transduction and gene control: the cAMP pathway. Crit Rev Oncog 3:321-338

17. Karolczak M, Korf HW, Stehle JH (2005) The rhythm and blues of gene expression in the rodent pineal gland. Endocrine 27:89100

18. Stehle JH, von Gall C, Korf HW (2001) Analysis of cell signalling in the rodent pineal gland deciphers regulators of dynamic transcription in neural/endocrine cells. Eur J Neurosci $14: 1-9$

19. Foulkes NS, Borjigin J, Snyder SH, Sassone-Corsi P (1997) Rhythmic transcription: the molecular basis of circadian melatonin synthesis. Trends Neurosci 20:487-492
20. Mouravlev A, Dunning J, Young D, During MJ (2006) Somatic gene transfer of cAMP response element-binding protein attenuates memory impairment in aging rats. Proc Natl Acad Sci U S A 103:4705-4710

21. Kojima N, Borlikova G, Sakamoto T, Yamada K, Ikeda T, Itohara S, Niki H, Endo S (2008) Inducible cAMP early repressor acts as a negative regulator for kindling epileptogenesis and long-term fear memory. J Neurosci 28:6459-6472

22. Laoide BM, Foulkes NS, Schlotter F, Sassone-Corsi P (1993) The functional versatility of CREM is determined by its modular structure. EMBO J 12:1179-1191

23. Foulkes NS, Borrelli E, Sassone-Corsi P (1991) CREM gene: use of alternative DNA-binding domains generates multiple antagonists of cAMP-induced transcription. Cell 64:739-749

24. Liu Y, Kalintchenko N, Sassone-Corsi P, Aguilera G (2006) Inhibition of corticotrophin-releasing hormone transcription by inducible cAMP-early repressor in the hypothalamic cell line, 4B. J Neuroendocrinol 18:42-49

25. Shepard JD, Liu Y, Sassone-Corsi P, Aguilera G (2005) Role of glucocorticoids and cAMP-mediated repression in limiting corticotropin-releasing hormone transcription during stress. J Neurosci 25:4073-4081

26. Mioduszewska B, Jaworski J, Kaczmarek L (2003) Inducible cAMP early repressor (ICER) in the nervous system-a transcriptional regulator of neuronal plasticity and programmed cell death. J Neurochem 87:1313-1320

27. Sassone-Corsi P (1998) Coupling gene expression to cAMP signalling: role of CREB and CREM. Int J Biochem Cell Biol 30:27-38

28. Monaco L, Sassone-Corsi P (1997) Cross-talk in signal transduction: Ras-dependent induction of cAMP-responsive transcriptional repressor ICER by nerve growth factor. Oncogene 15:2493-2500

29. Lund IV, Hu Y, Raol YH, Benham RS, Faris R, Russek SJ, Brooks-Kayal AR (2008) BDNF selectively regulates GABAA receptor transcription by activation of the JAK/STAT pathway. Sci Signal 1(4):ra9

30. Folco EJ, Koren G (1997) Degradation of the inducible cAMP early repressor (ICER) by the ubiquitin-proteasome pathway. Biochem J 328(Pt 1):37-43

31. Staiger JF, Bisler S, Schleicher A, Gass P, Stehle JH, Zilles K (2000) Exploration of a novel environment leads to the expression of inducible transcription factors in barrel-related columns. Neuroscience 99:7-16

32. Bisler S, Schleicher A, Gass P, Stehle JH, Zilles K, Staiger JF (2002) Expression of c-Fos, ICER, Krox-24 and JunB in the whisker-to-barrel pathway of rats: time course of induction upon whisker stimulation by tactile exploration of an enriched environment. J Chem Neuroanat 23:187-198

33. Kell CA, Dehghani F, Wicht H, Molina CA, Korf HW, Stehle JH (2004) Distribution of transcription factor inducible cyclic AMP early repressor (ICER) in rodent brain and pituitary. J Comp Neurol 478:379-394

34. Tinti C, Conti B, Cubells JF, Kim KS, Baker H, Joh TH (1996) Inducible cAMP early repressor can modulate tyrosine hydroxylase gene expression after stimulation of cAMP synthesis. J Biol Chem 271:25375-25381

35. Hu Y, Lund IV, Gravielle MC, Farb DH, Brooks-Kayal AR, Russek SJ (2008) Surface expression of GABAA receptors is transcriptionally controlled by the interplay of cAMP-response element-binding protein and its binding partner inducible cAMP early repressor. J Biol Chem 283:9328-9340

36. Chang JH, Vuppalanchi D, van Niekerk E, Trepel JB, Schanen NC, Twiss JL (2006) PC12 cells regulate inducible cyclic AMP (cAMP) element repressor expression to differentially control 
cAMP response element-dependent transcription in response to nerve growth factor and cAMP. J Neurochem 99:1517-1530

37. Fass DM, Butler JE, Goodman RH (2003) Deacetylase activity is required for cAMP activation of a subset of CREB target genes. J Biol Chem 278:43014-43019

38. Fitzgerald LR, Vaidya VA, Terwilliger RZ, Duman RS (1996) Electroconvulsive seizure increases the expression of CREM (cyclic AMP response element modulator) and ICER (inducible cyclic AMP early repressor) in rat brain. J Neurochem 66:429432

39. Konopka D, Szklarczyk AW, Filipkowski RK, Trauzold A, Nowicka D, Hetman M, Kaczmarek L (1998) Plasticity- and neurodegeneration-linked cyclic-AMP responsive element modulator/inducible cyclic-AMP early repressor messenger RNA expression in the rat brain. Neuroscience 86:499-510

40. Porter BE, Lund IV, Varodayan FP, Wallace RW, Blendy JA (2008) The role of transcription factors cyclic-AMP responsive element modulator (CREM) and inducible cyclic-AMP early repressor (ICER) in epileptogenesis. Neuroscience 152:829-836

41. Storvik M, Linden AM, Kontkanen O, Lakso M, Castren E, Wong G (2000) Induction of cAMP response element modulator (CREM) and inducible cAMP early repressor (ICER) expression in rat brain by uncompetitive N-methyl-D-aspartate receptor antagonists. J Pharmacol Exp Ther 294:52-60

42. Storvik M, Tiikkainen P, van Iersel M, Wong G (2006) Distinct gene expression profiles in adult rat brains after acute MK-801 and cocaine treatments. Eur Neuropsychopharmacol 16:211-219

43. Toronen P, Storvik M, Linden AM, Kontkane O, Marvanova M, Lakso M, Castren E, Wong G (2002) Expression profiling to understand actions of NMDA/glutamate receptor antagonists in rat brain. Neurochem Res 27:1209-1220

44. Della Fazia MA, Servillo G, Foulkes NS, Sassone-Corsi P (1998) Stress-induced expression of transcriptional repressor ICER in the adrenal gland. FEBS Lett 434:33-36

45. Mazzucchelli C, Sassone-Corsi P (1999) The inducible cyclic adenosine monophosphate early repressor (ICER) in the pituitary intermediate lobe: role in the stress response. Mol Cell Endocrinol 155:101-113

46. Green TA, Alibhai IN, Hommel JD, DiLeone RJ, Kumar A, Theobald DE, Neve RL, Nestler EJ (2006) Induction of inducible cAMP early repressor expression in nucleus accumbens by stress or amphetamine increases behavioral responses to emotional stimuli. J Neurosci 26:8235-8242

47. Conti AC, Kuo YC, Valentino RJ, Blendy JA (2004) Inducible cAMP early repressor regulates corticosterone suppression after tricyclic antidepressant treatment. J Neurosci 24:1967-1975

48. Gottlieb HB, Fleming TM, Ji L, Cunningham JT (2007) Identification of central nervous system sites involved in the water diuresis response elicited by central microinjection of nociceptin/Orphanin FQ in conscious rats via c-Fos and inducible cAMP early repressor immunocytochemistry. J Neuroendocrinol 19:531-542

49. Spencer CM, Houpt TA (2001) Dynamics of c-fos and ICER mRNA expression in rat forebrain following lithium chloride injection. Brain Res Mol Brain Res 93:113-126

50. Spencer CM, Jahng JW, Ryu V, Houpt TA (2005) Lithiuminduced gene expression of inducible cyclic adenosine monophosphate early repressor in the rat adrenal gland. J Neurosci Res $82: 273-282$

51. Luckman SM, Cox HJ (1995) Expression of inducible cAMP early repressor (ICER) in hypothalamic magnocellular neurons. Brain Res Mol Brain Res 34:231-238

52. Stehle JH, Pfeffer M, Kuhn R, Korf HW (1996) Light-induced expression of transcription factor ICER (inducible cAMP early repressor) in rat suprachiasmatic nucleus is phase-restricted. Neurosci Lett 217:169-172

53. Pfeffer M, Maronde E, Molina CA, Korf HW, Stehle JH (1999) Inducible cyclic AMP early repressor protein in rat pinealocytes: a highly sensitive natural reporter for regulated gene transcription. Mol Pharmacol 56:279-289

54. Maronde E, Pfeffer M, Olcese J, Molina CA, Schlotter F, Dehghani F, Korf HW, Stehle JH (1999) Transcription factors in neuroendocrine regulation: rhythmic changes in pCREB and ICER levels frame melatonin synthesis. J Neurosci 19:33263336

55. Misund K, Steigedal TS, Laegreid A, Thommesen L (2007) Inducible cAMP early repressor splice variants ICER I and IIgamma both repress transcription of c-fos and chromogranin A. J Cell Biochem 101:1532-1544

56. Mouravlev A, Young D, During MJ (2007) Phosphorylationdependent degradation of transgenic CREB protein initiated by heterodimerization. Brain Res 1130:31-37

57. Ho AK, Terriff DL, Price DM, Wloka MT, Chik CL (2007) The role of inducible repressor proteins in the adrenergic induction of arylalkylamine-N-acetyltransferase and mitogen-activated protein kinase phosphatase-1 in rat pinealocytes. Endocrinology 148:743-751

58. Foulkes NS, Borjigin J, Snyder SH, Sassone-Corsi P (1996) Transcriptional control of circadian hormone synthesis via the CREM feedback loop. Proc Natl Acad Sci U S A 93:14140 14145

59. Jaworski J, Mioduszewska B, Sanchez-Capelo A, Figiel I, Habas A, Gozdz A, Proszynski T, Hetman M, Mallet J, Kaczmarek L (2003) Inducible cAMP early repressor, an endogenous antagonist of cAMP responsive element-binding protein, evokes neuronal apoptosis in vitro. J Neurosci 23:4519-4526

60. Klejman A, Kaczmarek L (2006) Inducible cAMP early repressor (ICER) isoforms and neuronal apoptosis in cortical in vitro culture. Acta Neurobiol Exp (Wars) 66:267-272

61. Ruchaud S, Seite P, Foulkes NS, Sassone-Corsi P, Lanotte M (1997) The transcriptional repressor ICER and cAMP-induced programmed cell death. Oncogene 15:827-836

62. Mioduszewska B, Jaworski J, Szklarczyk AW, Klejman A, Kaczmarek L (2008) Inducible cAMP early repressor (ICER)evoked delayed neuronal death in the organotypic hippocampal culture. J Neurosci Res 86:61-70

63. Rami A, Bechmann I, Stehle JH (2008) Exploiting endogenous anti-apoptotic proteins for novel therapeutic strategies in cerebral ischemia. Prog Neurobiol 85:273-296

64. Tomita H, Nazmy M, Kajimoto K, Yehia G, Molina CA, Sadoshima J (2003) Inducible cAMP early repressor (ICER) is a negative-feedback regulator of cardiac hypertrophy and an important mediator of cardiac myocyte apoptosis in response to beta-adrenergic receptor stimulation. Circ Res 93:12-22

65. Maldonado R, Smadja C, Mazzucchelli C, Sassone-Corsi P (1999) Altered emotional and locomotor responses in mice deficient in the transcription factor CREM. Proc Natl Acad Sci U S A 96:14094-14099

66. Nemeroff CB, Widerlov E, Bissette G, Walleus H, Karlsson I, Eklund K, Kilts CD, Loosen PT, Vale W (1984) Elevated concentrations of CSF corticotropin-releasing factor-like immunoreactivity in depressed patients. Science 226:1342-1344

67. Bradley RG, Binder EB, Epstein MP, Tang Y, Nair HP, Liu W, Gillespie CF, Berg T, Evces M, Newport DJ, Stowe ZN, Heim CM, Nemeroff CB, Schwartz A, Cubells JF, Ressler KJ (2008) Influence of child abuse on adult depression: moderation by the corticotropin-releasing hormone receptor gene. Arch Gen Psychiatry $65: 190-200$ 
68. Cain DP (1989) Long-term potentiation and kindling: how similar are the mechanisms? Trends Neurosci 12:6-10

69. Barnes SJ, Pinel JP (2001) Conditioned effects of kindling. Neurosci Biobehav Rev 25:745-751

70. Bading H (1999) Nuclear calcium-activated gene expression: possible roles in neuronal plasticity and epileptogenesis. Epilepsy Res 36:225-231

71. Cain DP, Corcoran ME, Staines WA (1980) Effects of protein synthesis inhibition on kindling in the mouse. Exp Neurol 68:409-419

72. Goddard GV (1967) Development of epileptic seizures through brain stimulation at low intensity. Nature 214:1020-1021

73. Kashihara K, Sato K, Akiyama K, Ishihara T, Hayabara T, Abe K (2000) Temporal profile of CRE DNA-binding activity in the rat hippocampus following a kindling stimulation. Epilepsy Res 40:171-177

74. Lopez de Armentia M, Jancic D, Olivares R, Alarcon JM, Kandel ER, Barco A (2007) cAMP response element-binding protein-mediated gene expression increases the intrinsic excitability of CA1 pyramidal neurons. J Neurosci 27: 13909-13918

75. Bourtchuladze R, Frenguelli B, Blendy J, Cioffi D, Schutz G, Silva AJ (1994) Deficient long-term memory in mice with a targeted mutation of the cAMP-responsive element-binding protein. Cell 79:59-68

76. Schulz S, Siemer H, Krug M, Hollt V (1999) Direct evidence for biphasic cAMP responsive element-binding protein phosphorylation during long-term potentiation in the rat dentate gyrus in vivo. J Neurosci 19:5683-5692

77. Barco A, Alarcon JM, Kandel ER (2002) Expression of constitutively active CREB protein facilitates the late phase of long-term potentiation by enhancing synaptic capture. Cell 108:689-703

78. Yin JC, Wallach JS, Del Vecchio M, Wilder EL, Zhou H, Quinn WG, Tully T (1994) Induction of a dominant negative CREB transgene specifically blocks long-term memory in Drosophila. Cell 79:49-58

79. Bartsch D, Ghirardi M, Skehel PA, Karl KA, Herder SP, Chen M, Bailey CH, Kandel ER (1995) Aplysia CREB2 represses long-term facilitation: relief of repression converts transient facilitation into long-term functional and structural change. Cell 83:979-992

80. Josselyn SA, Nguyen PV (2005) CREB, synapses and memory disorders: past progress and future challenges. Curr Drug Targets CNS Neurol Disord 4:481-497

81. Balschun D, Wolfer DP, Gass P, Mantamadiotis T, Welzl H, Schutz G, Frey JU, Lipp HP (2003) Does cAMP response element-binding protein have a pivotal role in hippocampal synaptic plasticity and hippocampus-dependent memory? J Neurosci 23:6304-6314

82. Kogan JH, Frankland PW, Blendy JA, Coblentz J, Marowitz Z, Schutz G, Silva AJ (1997) Spaced training induces normal longterm memory in CREB mutant mice. Curr Biol 7:1-11

83. Josselyn SA, Kida S, Silva AJ (2004) Inducible repression of CREB function disrupts amygdala-dependent memory. Neurobiol Learn Mem 82:159-163

84. Gass P, Wolfer DP, Balschun D, Rudolph D, Frey U, Lipp HP, Schutz G (1998) Deficits in memory tasks of mice with CREB mutations depend on gene dosage. Learn Mem 5:274-288

85. Blendy JA, Kaestner KH, Schmid W, Gass P, Schutz G (1996) Targeting of the CREB gene leads to up-regulation of a novel CREB mRNA isoform. EMBO J 15:1098-1106

86. Hummler E, Cole TJ, Blendy JA, Ganss R, Aguzzi A, Schmid W, Beermann F, Schutz G (1994) Targeted mutation of the
CREB gene: compensation within the CREB/ATF family of transcription factors. Proc Natl Acad Sci U S A 91:5647-5651

87. Sakai N, Thome J, Newton SS, Chen J, Kelz MB, Steffen C, Nestler EJ, Duman RS (2002) Inducible and brain regionspecific CREB transgenic mice. Mol Pharmacol 61:1453-1464

88. Machado HB, Vician LJ, Herschman HR (2008) The MAPK pathway is required for depolarization-induced "promiscuous" immediate-early gene expression but not for depolarizationrestricted immediate-early gene expression in neurons. J Neurosci Res 86:593-602

89. Kida S, Josselyn SA, de Ortiz SP, Kogan JH, Chevere I, Masushige S, Silva AJ (2002) CREB required for the stability of new and reactivated fear memories. Nat Neurosci 5:348355

90. Brightwell JJ, Smith CA, Countryman RA, Neve RL, Colombo PJ (2005) Hippocampal overexpression of mutant creb blocks long-term, but not short-term memory for a socially transmitted food preference. Learn Mem 12:12-17

91. Pittenger C, Huang YY, Paletzki RF, Bourtchouladze R, Scanlin H, Vronskaya S, Kandel ER (2002) Reversible inhibition of CREB/ATF transcription factors in region CA1 of the dorsal hippocampus disrupts hippocampus-dependent spatial memory. Neuron 34:447-462

92. Josselyn SA, Shi C, Carlezon WA Jr, Neve RL, Nestler EJ, Davis M (2001) Long-term memory is facilitated by cAMP response element-binding protein overexpression in the amygdala. J Neurosci 21:2404-2412

93. Brightwell JJ, Smith CA, Neve RL, Colombo PJ (2007) Longterm memory for place learning is facilitated by expression of cAMP response element-binding protein in the dorsal hippocampus. Learn Mem 14:195-199

94. Wallace TL, Stellitano KE, Neve RL, Duman RS (2004) Effects of cyclic adenosine monophosphate response element binding protein overexpression in the basolateral amygdala on behavioral models of depression and anxiety. Biol Psychiatry $56: 151-160$

95. Brightwell JJ, Smith CA, Neve RL, Colombo PJ (2008) Transfection of mutant CREB in the striatum, but not the hippocampus, impairs long-term memory for response learning. Neurobiol Learn Mem 89:27-35

96. Yuan Q, Harley CW, Darby-King A, Neve RL, McLean JH (2003) Early odor preference learning in the rat: bidirectional effects of cAMP response element-binding protein (CREB) and mutant CREB support a causal role for phosphorylated CREB. J Neurosci 23:4760-4765

97. Carlezon WA Jr, Duman RS, Nestler EJ (2005) The many faces of CREB. Trends Neurosci 28:436-445

98. Pandey SC, Chartoff EH, Carlezon WA Jr, Zou J, Zhang H, Kreibich AS, Blendy JA, Crews FT (2005) CREB gene transcription factors: role in molecular mechanisms of alcohol and drug addiction. Alcohol Clin Exp Res 29:176-184

99. Nestler EJ (2004) Molecular mechanisms of drug addiction. Neuropharmacology 47(Suppl 1):24-32

100. Blendy JA, Maldonado R (1998) Genetic analysis of drug addiction: the role of cAMP response element binding protein. $\mathrm{J}$ Mol Med 76:104-110

101. Walters CL, Kuo YC, Blendy JA (2003) Differential distribution of CREB in the mesolimbic dopamine reward pathway. J Neurochem 87:1237-1244

102. Carlezon WA Jr, Thome J, Olson VG, Lane-Ladd SB, Brodkin ES, Hiroi N, Duman RS, Neve RL, Nestler EJ (1998) Regulation of cocaine reward by CREB. Science 282:2272-2275

103. Pliakas AM, Carlson RR, Neve RL, Konradi C, Nestler EJ, Carlezon WA Jr (2001) Altered responsiveness to cocaine and 
increased immobility in the forced swim test associated with elevated cAMP response element-binding protein expression in nucleus accumbens. J Neurosci 21:7397-7403

104. Ishiguro H, Kim KT, Joh TH, Kim KS (1993) Neuron-specific expression of the human dopamine beta-hydroxylase gene requires both the cAMP-response element and a silencer region. J Biol Chem 268:17987-17994

105. Kim KS, Lee MK, Carroll J, Joh TH (1993) Both the basal and inducible transcription of the tyrosine hydroxylase gene are dependent upon a cAMP response element. J Biol Chem 268:15689-15695

106. Lamas M, Sassone-Corsi P (1997) The dynamics of the transcriptional response to cyclic adenosine 3', 5'-monophos- phate: recurrent inducibility and refractory phase. Mol Endocrinol $11: 1415-1424$

107. Won J, Silva AJ (2008) Molecular and cellular mechanisms of memory allocation in neuronetworks. Neurobiol Learn Mem 89 (3):285-292

108. Abraham WC, Bear MF (1996) Metaplasticity: the plasticity of synaptic plasticity. Trends Neurosci 19:126-130

109. Morgan JI, Curran T (1991) Stimulus-transcription coupling in the nervous system: involvement of the inducible protooncogenes fos and jun. Annu Rev Neurosci 14:421-451

110. Sheng M, Greenberg ME (1990) The regulation and function of c-fos and other immediate early genes in the nervous system. Neuron 4:477-485 